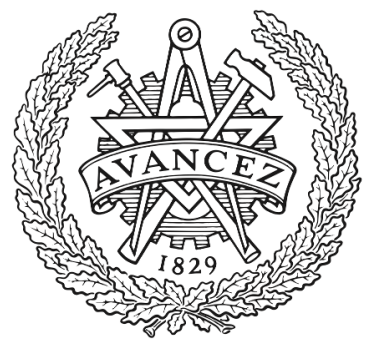

CHALMERS

UNIVERSITY OF TECHNOLOGY

\title{
Comparison of uniform cross QAM and probabilistically shaped QAM formats under the impact of transmitter impairments
}

Downloaded from: https://research.chalmers.se, 2023-04-26 13:03 UTC

Citation for the original published paper (version of record):

Mirani, A., Mazur, M., Agrell, E. et al (2019). Comparison of uniform cross QAM and probabilistically shaped QAM formats under the impact of transmitter impairments. IET Conference Publications(CP765). http://dx.doi.org/10.1049/cp.2019.0994

N.B. When citing this work, cite the original published paper. 


\title{
COMPARISON OF UNIFORM CROSS QAM AND PROBABILISTICALLY SHAPED QAM FORMATS UNDER THE IMPACT OF TRANSMITTER IMPAIRMENTS

\author{
Ali Mirani ${ }^{1,2 *}$, Mikael Mazur ${ }^{1}$, Erik Agrell ${ }^{2}$, Benjamin Foo ${ }^{1}$, Jochen Schröder ${ }^{1}$, \\ Peter A. Andrekson ${ }^{1}$, Magnus Karlsson ${ }^{1}$
}

${ }^{I}$ Photonics Laboratory, Department of Microtechnology and Nanoscience, Chalmers University of Technology, Gothenburg, Sweden

${ }^{2}$ Department of Electrical Engineering, Chalmers University of Technology, Gothenburg, Sweden

*E-mail:mirani@chalmers.se

\begin{abstract}
Keywords: OPTICAL FIBER COMMUNICATION, TRANSCEIVER IMPAIRMENTS, PROBABILISTIC SHAPING, ACHIEVABLE INFORMATION RATE, SYMBOL ERROR RATE
\end{abstract}

\begin{abstract}
Considering the nonideal response of Mach-Zehnder modulators, uniform cross QAM constellations improve upon probabilistically shaped QAM by a factor of up to 4 in uncoded symbol error rate and also offer higher achievable information rates, at the same source entropy and optimal electrical signal powers.
\end{abstract}

\section{Introduction}

Communication systems are evolving towards using the maximum information transfer rate and approaching the capacity bounds for every specific channel [1]. One of the promising methods to approach the capacity limit is to shape the input signal constellation to mimic a certain distribution which maximizes the capacity of the link [2]. For the additive white Gaussian noise (AWGN) channel, this distribution is known to be Gaussian and there is a $1.53 \mathrm{~dB}$ gap between the capacity limit and what can be achieved by the conventional uniformly distributed equidistant constellations [3]. Two main directions to shape the input signal constellation are probabilistic shaping (PS) and geometric shaping [4]. Both of these techniques will increase the power efficiency, transmission reach, and tolerance to system impairments [4-6]. One of the advantages of PS is that it can be used in the current equidistant quadrature amplitude modulation (QAM) systems using similar symbol-wise coherent digital signal processing (DSP) [7].

One factor limiting current transmission systems is impairments caused by the transceivers [8]. The non-ideal transfer functions of these components will modify the signal quality depending on the driving signal amplitude and bandwidth. Digital-to-analog converter (DAC), Mach-Zehnder modulator (MZM), optical frontend and analog-to-digital converter (ADC) are components with bandwidth limitations and are often modeled as having a low-pass response [8]. According to [8], in order to upgrade the systems to higher order modulation formats, the bandwidth requirements for the transmitter side components are higher than on the receiver side. Therefore, we focus on the transmitter impairments and neglect the receiver components' constraints.

In this paper, the impact of limitations induced by the DAC and MZM on the performance of the uniform cross-shaped 32QAM (Uni-32QAM) and PS-64QAM with the same source entropy is studied. The impairments include the bandwidth limitation, quantization noise and the sinusoidal transfer function of the MZM with respect to driving voltage. The achievable information rate (AIR) and symbol error rate (SER) are calculated to compare the performance for these modulation formats. It is known that in linear AWGN channel, PS constellations perform better than the uniform constellations [9]. However, we find that considering the transmitter impairments, Uni-32QAM outperforms PS-64QAM by optimizing the electrical signal power to the MZM.

\section{AIR and SER calculation}

The mutual information (MI) between two random vectors of $\underline{X}$ and $\underline{Y}$ is defined as [3]

$$
I(\underline{X} ; \underline{Y})=\mathbb{E}_{\underline{X}, \underline{Y}}\left[\log _{2} \frac{p_{\underline{Y} \mid \underline{X}}(\underline{Y} \mid \underline{X})}{p_{\underline{Y}}(\underline{Y})}\right],
$$

where $\mathbb{E}_{\underline{X}, \underline{Y}}$ denotes the expectation with respect to both $\underline{X}$ and $\underline{Y}, p_{\underline{Y} \mid \underline{X}}(\underline{y} \mid \underline{x})$ is the conditional probability distribution between $\underline{X}$ and $\underline{Y}$, and $p_{\underline{Y}}(y)$ is the joint probability distribution function of the random vector $Y$.

In most optical communications systems, it is assumed that DSP at the receiver can compensate the effects of the channel memories to a large extent [3], [10]. Therefore, instead of dependent random variables as a vector $(\underline{Y})$, we can limit the calculations to independent and identically distributed (i.i.d.) random variables $(Y)$. Using the weak law of large numbers [11] and approximating the actual conditional distribution $p_{Y \mid X}(y \mid x)$ by a suboptimal auxiliary channel model $\left(q_{Y \mid X}(y \mid x)\right)$, (1) can be lower bounded by $K$ symbol pairs of these random variables and taking the average over these realizations as

$$
I(X ; Y) \geq \frac{1}{K} \sum_{i=1}^{K}\left[\log _{2} \frac{q_{Y \mid X}\left(y_{i} \mid x_{i}\right)}{q_{Y}\left(y_{i}\right)}\right],
$$




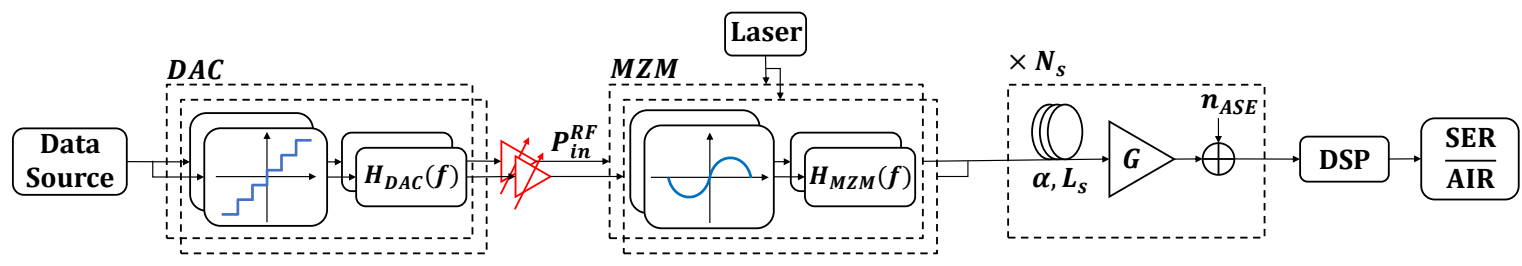

Fig. 1. Simulation setup including the transmitter impairments.

if $K$ is large enough.

In this work, $q_{Y \mid X}(y \mid x)$ is considered to be an AWGN channel with discrete input random variable $X$ and continuous output random variable $Y$, which are related by $Y=X+Z$ where $Z$ is a complex, zero-mean, circularly symmetric Gaussian random variable. The auxiliary channel law is assumed to be

$$
q_{Y \mid X}(y \mid x)=\frac{1}{2 \pi \sigma^{2}} \exp \left(-\frac{|y-x|^{2}}{2 \sigma^{2}}\right),
$$

and $q_{Y}(y)=\sum_{x \in \mathcal{X}} q_{Y \mid X}(y \mid x) p_{X}(x)$, where $2 \sigma^{2}$ indicates the total power of the complex noise, $\mathcal{X}$ is the source alphabet, and $p_{X}(x)$ is the input distribution over alphabet.

For probabilistically shaped or uniform constellation formats, in order to calculate the SER, the maximum a posteriori (MAP) decoding rule has been applied. For a channel with the auxiliary channel law described by (3), the symbol decision rule is

$$
\hat{x}=\arg \min _{x}\left[|y-x|^{2}+2 \sigma^{2} \cdot \log _{\mathrm{e}}\left(\frac{2 \pi \sigma^{2}}{p_{X}(x)}\right)\right],
$$

which generalizes the conventional maximum likelihood (minimum distance) detector to nonuniform constellations.

\section{Simulation setup}

In the simulation setup, receiver limitations, dispersion and nonlinearity of the fiber link are ignored and we have focused on the transmitter constraints. The parameters of the simulation are listed at Tab. 1.

As shown in Fig. 1, the simulations setup includes a source which generates complex QAM symbols at the fixed symbol rate of $28 \mathrm{GSym} / \mathrm{s}$ for all of the modulation formats [12]. The performance and simulation setup blocks over each quadrature are assumed to be identical. To estimate each AIR or SER value, we generated more than $10^{5}$ symbols. In order to generate PS-64QAM constellation points, we shaped the probability distribution of the symbols by the MaxwellBoltzmann distribution [7] to have a source entropy equal to 5 bits/symbol in order to be comparable with Uni-32QAM. The probability of symbol $x_{i}$ is $p_{X}\left(x_{i}\right)=e^{-\nu\left|x_{i}\right|^{2}} / \sum_{x \in \mathcal{X}} e^{-\nu|x|^{2}}$ where $\nu$ is the shaping factor. The shaping factor was kept fixed in this work to have an equal source entropy compared with the uniform cross QAM format. A demonstration of the constellation formats in this setup is shown in Fig. 2. Next, the vector of symbols is upsampled and filtered by a root-raised cosine (RRC) filter with the roll-off factor of 0.01 to perform pulse shaping, reducing the side lobe level and interference. In the next step, the signal amplitude is normalized in each

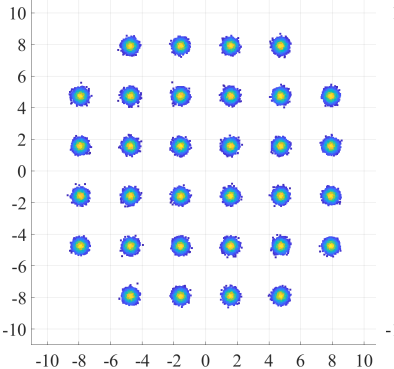

(a)

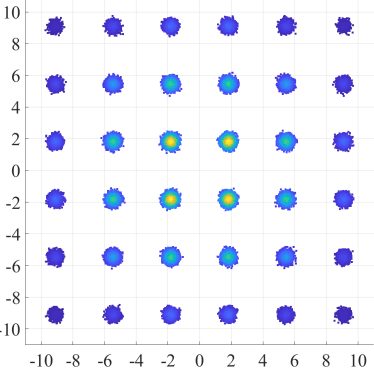

(b)
Fig. 2 Amplitude comparison of (a) Uni-32QAM and (b) PS-64QAM at the same electrical signal power and same AWGN power.

quadrature, quantized depending on the resolution of the DAC and filtered out by a second order Bessel filter $\left(H_{D A C}(f)\right)$ [8], [12]. The electric output signal of the DAC is amplified to drive the MZM which is connected to a $10 \mathrm{dBm}$ laser at 1550 $\mathrm{nm}$. The MZM is considered to have a sinusoidal transfer function with respect to driving voltage followed by a Gaussian transfer function with respect to frequency $\left(H_{M Z M}(f)\right)$ [8]. The optically modulated signal at the output of the MZM is connected to a linear channel consisting of $80 \mathrm{~km}$ fiber spans and in-line amplifiers to compensate the fiber losses [13].

In the receiver, the effect of the limited bandwidth of the transmitter components is compensated using their corresponding matched filter and the signal is downsampled to retrieve the received symbols in order to calculate the desired performance metric

\section{Results}

We evaluate the performance of Uni-32QAM and PS-64QAM with source entropy 5 bits/sym in simulations using (2), (4) and the parameters in Tab. 1, and we compare the AIR and SER for these modulation formats.

Fig. 3 shows the SER comparison between the Uni-32QAM and PS-64QAM considering a linear modulator and an MZM

Table 1 Simulation parameters

\begin{tabular}{llll}
\hline Parameter & Value & Parameter & Value \\
\hline DAC bandwidth & $18 \mathrm{GHz}$ & DAC resolution & $8 \mathrm{bits}$ \\
MZM bandwidth & $25 \mathrm{GHz}$ & MZM $V_{\pi}, \mathrm{R}_{\mathrm{L}}$ & $5 \mathrm{~V}, 50 \Omega$ \\
MZM insertion loss & $5 \mathrm{~dB}$ & Laser power & $10 \mathrm{dBm}$ \\
Roll-off factor & 0.01 & Oversampling rate & 2 \\
EDFA Noise figure & $6 \mathrm{~dB}$ & Span loss & $16 \mathrm{~dB}$ \\
\hline
\end{tabular}


with a sinusoidal response. Different groups of curves correspond to different transmission distances, which in this model scale linearly with the AWGN power. For the ideal linear modulator, the results follow the same trend as an AWGN channel and PS-64QAM outperforms Uni-32QAM in terms of SER for every input electrical signal power. This is because of the larger minimum distance between the constellation points of PS-64QAM than Uni-32QAM at the same power as shown in Fig. 2. However, the situation changes when the sinusoidal response of the MZM is taken into account.

As shown in Fig. 3, there is an optimum power for SER at every transmission distance for each of the modulation formats. For low AWGN power, the optimum SER values are better for Uni-32QAM. Fig. 3 shows that Uni-32QAM reduces the uncoded SER over PS-64QAM by up to a factor of 4 at optimal powers for the considered transmission distances. The reason for this effect can be described based on Fig. 2. At the same input electrical signal power to the MZM, the maximum signal amplitude is smaller for Uni32QAM compared with PS-64QAM. Therefore, at the same electrical signal power, while the PS-64QAM amplitude is entering the nonlinear region of the MZM input swing, the power of Uni-32QAM can still be increased without passing into the nonlinear amplitude region of the MZM. Hence, by remaining in the linear region, the SER will decrease more until the amplitude of the signal becomes comparable to $V_{\pi}$ of the MZM for Uni-32QAM. For high values of AWGN power and high SER (long transmission distances), PS-64QAM performs better than Uni-32QAM and this because the AWGN dominates the nonlinear effects of the transmitter.

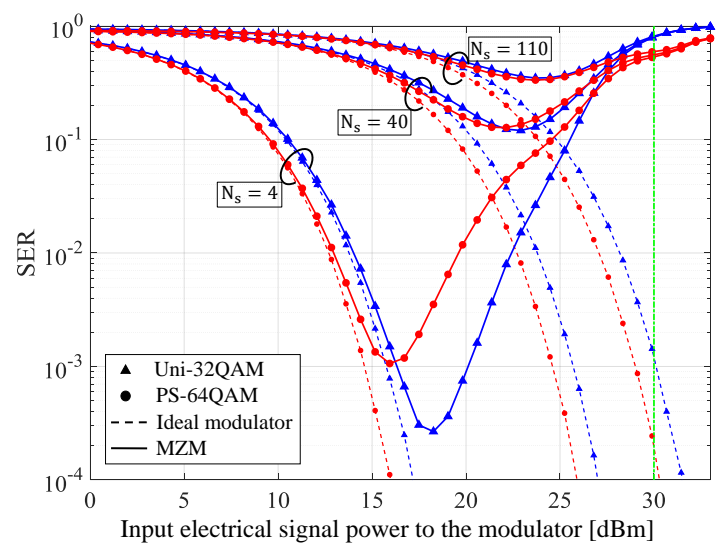

Fig. 3 SER vs. input electrical signal power to a modulator. The green line is the equivalent power for a square signal with $-\mathrm{V}_{\pi}$ and $\mathrm{V}_{\pi}$ swing.

In Fig. 4, an AIR comparison, for the same conditions as in Fig. 3, is shown. At low AWGN power, both modulation formats saturate to their maximum source entropy. By increasing the transmission distance, there will be a maximum AIR for each modulation format. Simulations show that the maximum AIR is higher for Uni-32QAM and the gap between these maximums increases at higher noise powers.

In [14] and [15], it was shown that PS outperforms uniform modulation formats for different transmission distances.

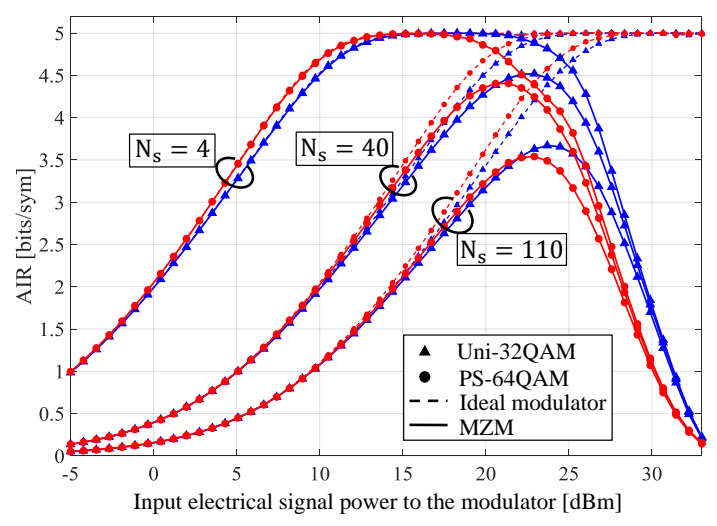

Fig. 4 AIR vs. input electrical signal power to the modulator.

In this work, we found the opposite behavior for our channel model including the transmitter impairments. Fig. 5 shows that, by optimizing the input electrical signal power to the MZM, Uni-32QAM and Uni-128QAM perform better in terms of AIR. However, if the input electrical signal power to the MZM is kept fixed, e.g., optimized for the back-toback setup without AWGN, the result will change and PS modulation format will perform better as reported in [14] and [15].

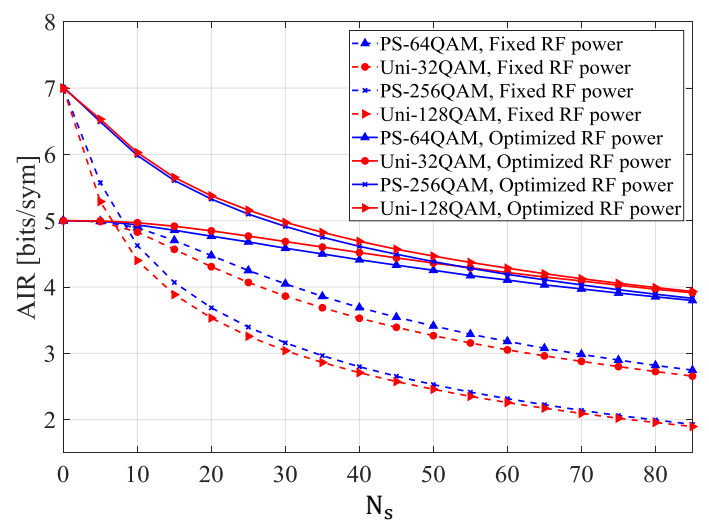

Fig. 5 AIR vs. transmission distance for optimized and fixed input electrical signal power to the MZM.

\section{Conclusion}

In contrast with the improved performance of PS modulation formats over uniform modulation formats in linear channels, we have demonstrated that optimizing the input electrical signal power to the MZM leads to a performance improvement of Uni-32QAM and Uni-128QAM with respect to PS-64QAM and PS-256QAM with same source entropy in a linear fiber channel with transmitter impairments. We have shown up to a factor of 4 improvement in the uncoded SER for Uni-32QAM over PS-64QAM in the range of studied parameters.

\section{Acknowledgements}

This work was supported by the Swedish Research Council under grant no. 2015-04239 and 2017-03702. 


\section{References}

[1] P. J. Winzer, D. T. Neilson, and A. R. Chraplyvy, "Fiberoptic transmission and networking: the previous 20 and the next 20 years," Optics Express, vol. 26, no. 18, pp. 24 190-24 239, 2018.

[2] C. E. Shannon, "A mathematical theory of communication," Bell system technical journal, vol. 27, no. 3, pp. 379-423, 1948.

[3] A. Alvarado, T. Fehenberger, B. Chen, and F. M. Willems, "Achievable information rates for fiber optics: Applications and computations," Journal of Lightwave Technology, vol. 36, no. 2, pp. 424-439, 2018.

[4] Z. Qu and I. B. Djordjevic, "On the probabilistic shaping and geometric shaping in optical communication systems," IEEE Access, vol. 7, pp. 21 454-21 464, 2019.

[5] F. Buchali, F. Steiner, G. Böcherer, L. Schmalen, P. Schulte, and W. Idler, "Rate adaptation and reach increase by probabilistically shaped 64-QAM: An experimental demonstration," Journal of Lightwave Technology, vol. 34, no. 7, pp. 1599-1609, 2016.

[6] M. P. Yankov, K. J. Larsen, and S. Forchhammer, "Temporal probabilistic shaping for mitigation of nonlinearities in optical fiber systems," Journal of Lightwave Technology, vol. 35, no. 10, pp. 1803-1810, 2017.

[7] J. Cho and P. Winzer, "Probabilistic constellation shaping for optical fiber communications," Journal of Lightwave Technology, 2019.

[8] D. Rafique, H. Griesser, and J.-P. Elbers, "Enabling 64Gbaud coherent optical transceivers," in 2017 Optical Fiber Communications Conference and Exhibition (OFC). IEEE, 2017.

[9] T. Fehenberger, A. Alvarado, G. Böcherer, and N. Hanik, "On probabilistic shaping of quadrature amplitude modulation for the nonlinear fiber channel," Journal of Lightwave Technology, vol. 34, no. 21, pp. 5063-5073, 2016.

[10] L. Szczecinski and A. Alvarado, Bit-interleaved coded modulation: fundamentals, analysis and design. John Wiley \& Sons, 2015.

[11] A. A. El-Rahman and J. Cartledge, "Multidimensional geometric shaping for QAM constellations," in 2017 European Conference on Optical Communication (ECOC). IEEE, 2017.

[12] D. Rafique, T. Rahman, A. Napoli, and B. Spinnler, "Digital pre-emphasis in optical communication systems: On the nonlinear performance," Journal of Lightwave Technology, vol. 33, no. 1, pp. 140-150, 2015.

[13] K.-P. Ho, Phase-modulated optical communication systems. Springer Science \& Business Media, 2005.

[14] F. Buchali, W. Idler, L. Schmalen, and Q. Hu, "Flexible optical transmission close to the shannon limit by probabilistically shaped QAM," in 2017 Optical Fiber Communications Conference and Exhibition (OFC). IEEE, 2017.

[15] S. Chandrasekhar, B. Li, J. Cho, X. Chen, E. Burrows, G. Raybon, and P. Winzer, "High-spectral-efficiency transmission of PDM 256-QAM with parallel probabilistic shaping at record rate-reach trade-offs," in
ECOC 2016-Post Deadline Paper; 42nd European Conference on Optical Communication. VDE, 2016. 